\title{
HOMOTOPY TREES WITH TRIVIAL CLASSIFYING RING
}

\author{
MICHEAL N. DYER
}

\begin{abstract}
AbSTRaCr. In this note we study a certain class of groups $\pi$ for which the homotopy classification of $(\pi, m)$-complexes is independent of the $k$-invariant for small $m$.
\end{abstract}

1. Homotopy trees. A $(\pi, m)$-complex is a finite, connected $m$-dimensional $\mathrm{CW}$-complex such that $\pi_{1}(X)=\pi$ and $\pi_{i}(X)=0$ for $1<i<m$. The homotopy tree $H T(\pi, m)$ is a directed tree whose vertices are homotopy classes of $(\pi, m)$-complexes. A vertex $[X]$ is connected by an edge to a vertex $[Y]$ iff $Y \cong X \vee S^{m}$. The problem is to describe $H T(\pi, m)$ (see [3]).

There is an algebraic analog to this problem in the theory of algebraic $m$ types. The algebraic $m$-type $\Pi(X)$ of a $(\pi, m)$-complex $X$ is a triple $\Pi(X)$ $=\left(\pi, \pi_{m}(X), k(X)\right)$ where $k(X) \in H^{m+1}\left(\pi, \pi_{m}(X)\right)$ is the first $k$-invariant of $X$ (see [7], [3]). It is proved in [4], that if $\pi_{m}=\pi_{m}(X)$ is finitely generated as a $\pi$-module and $H^{m+1}\left(\pi ; Z_{\pi}\right)=0$, then $R(\pi, m)=H^{m+1}\left(\pi, \pi_{m}\right)$ has the structure of a ring with identity such that the units $U(\pi, m)$ of $H^{m+1}\left(\pi, \pi_{m}\right)$ are the projective $k$-invariants. Note that $H^{m+1}\left(\pi, \pi_{m}(X)\right) \cong H^{m+1}\left(\pi, \pi_{m}(Y)\right)$ for any two $(\pi, m)$-complexes $X$ and $Y$, by Schanuel's lemma. In $\S 2$ we isolate a large class of groups $\pi$ having $H^{i}(\pi ; Z \pi)=0$ for all but one value of $i$.

Furthermore, there is a homomorphism $\kappa: U(\pi, m) \rightarrow \tilde{K}_{0} Z \pi$ where $\tilde{K}_{0} Z \pi$ is the reduced projective class group of the integral group ring $Z \pi$ of $\pi$. The kernel of $\kappa$, denoted $S F(\pi, m)$, is precisely the set of units which arise as the $k$ invariants of $(\pi, m)$-complexes.

DEFINITION. The ring $R(\pi, m)$ is called the classifying ring of the homotopy tree $H T(\pi, m) ; \kappa: U \rightarrow \tilde{K}_{0} Z \pi$, the classifying homomorphism.

We say that the classifying ring of $H T(\pi, m)$ is trivial if it is isomorphic to the zero ring or the ring of integers $Z$. In the latter case $\kappa: U \rightarrow \tilde{K}_{0} Z \pi$ is the zero homomorphism.

In order to state the results, we need to define an isomorphism of algebraic $m$-types. $(\theta, \beta): \Pi(X) \rightarrow \Pi(Y)$ is an isomorphism if $\theta: \pi \rightarrow \pi$ is a group automorphism, $\beta$ : $\pi_{m}(X) \rightarrow \pi_{m}(Y)$ is a $\theta$-isomorphism of $\pi$-modules $(\beta(x \cdot y)$ $=\theta(x) \beta(y)$, for $x \in \pi, y \in \pi_{m}(X)$ and $\beta$ is bijective) and $\theta^{*-1} \beta_{*}(k(X))$ $=k(Y)$ in the following diagram:

$$
H^{m+1}\left(\pi, \pi_{m}(X)\right) \stackrel{\beta^{*}}{\longrightarrow} H^{m+1}\left(\pi, \pi_{m}(Y)_{\theta}\right) \stackrel{\theta^{*}}{\stackrel{H^{m+1}}{\leftrightarrows}}\left(\pi, \pi_{m}(Y)\right)
$$

where $\pi_{m}(Y)_{\theta}$ is the $\pi$-module with action $x * y=\theta(x) \cdot y(x \in \pi, y$

Received by the editors May 9, 1975.

AMS (MOS) subject classifications (1970). Primary 55D15, $18 \mathrm{H} 10$. 
$\left.\in \pi_{m}(Y)\right)$.It is known from [7] that $X$ and $Y$ have the same homotopy type iff $\Pi(X) \cong \Pi(Y)$.

We say that the homotopy tree $H T(\pi, m)$ is independent of the $k$-invariant if any two $(\pi, m)$-complexes $X$ and $Y$ having $\theta$-isomorphic $(\theta \in$ Aut $\pi) m$ homotopy modules have the same homotopy type.

1.1. THEOREM. If the classifying ring $R(\pi, m)$ is trivial, then the homotopy tree $H T(\pi, m)$ is independent of the k-invariant.

Proof. For the proof we use the following result of S. Eilenberg and S. Mac Lane: for any $(\pi, m)$-complexes $X, Y$ such that $(\theta, \beta): \Pi(X) \cong \Pi(Y)$ there exists a map $f: X \rightarrow Y$ realizing $(\theta, \beta)$ in the sense that $f_{1 \#}=\theta$ and $f_{m \#}=\beta$.

The theorem is clear if $R(\pi, m)=0$. Now suppose that $H^{m+1}\left(\pi, \pi_{m}(X)\right)$ $\cong Z$ as a ring. Now $\beta_{*}^{-1} \circ \theta^{*}(1)= \pm 1$ because $\beta_{*}^{-1} \circ \theta^{*}$ is an isomorphism $Z \rightarrow Z$. Thus, as $k(X)$ and $k(Y)$ must be units in $Z$, one of

$$
(\theta, \pm \beta):\left(\pi, \pi_{m}(X), k(X)\right) \rightarrow\left(\pi, \pi_{m}(Y), \pm \theta^{*-1} \circ \beta_{*}(k(X))\right)
$$

is an isomorphism $\Pi(X) \rightarrow \Pi(Y)$. This completes (1.1).

We now consider a class of groups $\mathscr{P}^{n}(1<n \leqslant \infty)$. A group $G$ has property $\rho^{n}$ if $H^{i}(G ; Z G)=0$ for $0<i<n$. This is a rather large class. For example, any finite group has $\mathcal{P}^{\infty}$; finitely generated abelian groups of rank $n$ have $\mathcal{P}^{n}(n \geqslant 1)$ [2, Proposition 3.1]. Also, duality groups of cohomological dimension $n$ have $\mathscr{P}^{n}[1]$, [2], [6]. In $\S 2$, we study a restricted class $\bigodot$ which is closed under extensions. From this we see that infinite polycyclic groups having Hirsch number $n$ are $\mathcal{P}^{n}$-groups.

1.2. THEOREM. Let $n$ be an integer $\geqslant 4$. Let $\pi$ be an infinite group satisfying $\rho^{n}$ and let $m+1<n$. Then the classifying ring $R(\pi, m)$ is infinite cyclic.

Let $\xi(X, Y)$ denote the set of homotopy classes of homotopy equivalences $X \rightarrow Y ; \xi(X) \equiv \xi(X, X)$ the self-equivalence group of $X$.

1.3. Corollary. Under the above hypotheses, let $X, Y$ be two $(\pi, m)$-complexes having the same homotopy type. Then

(i) $\xi(X, Y) \cong \operatorname{Iso}(\Pi(X), \Pi(Y))$ (set bijection),

(ii) $\xi(X) \cong \operatorname{Aut}(\Pi(X))$ (group isomorphism). The isomorphisms are given by carrying $(f: X \rightarrow Y) \mapsto\left(f_{\#}: \Pi(X) \rightarrow \Pi(Y)\right)$.

A. J. Sieradski has proved the corollary for $\pi$ a finite group [3, Theorem 6.6].

Proof. Let $X$ be any $(\pi, m)$-complex. $H^{i}(\pi ; Z \pi)=0$ for $0<i \leqslant m+1$ implies that $H^{m+1}\left(\pi, \pi_{m}(X)\right) \cong H^{0}(\pi, Z) \cong Z$ as a ring. Also $H^{m}\left(\pi, \pi_{m}(X)\right)$ $\cong H^{0}(\pi ; A(\pi))=A(\pi)^{\pi}=0$ because $\pi$ is infinite. Here $A(\pi)$ is the augmentation ideal. The corollary follows from Theorem 6.4 of [3] because $H^{m}\left(\pi, \pi_{m}(X)\right)$ measures the number of homotopy classes of homotopy equivalences $f: X \rightarrow Y$ inducing the same isomorphism $f_{\#}: \Pi(X) \rightarrow \Pi(Y)$.

Two $\pi$-modules $M, m^{\prime}$ are $\theta$-isomorphic $(\theta \in$ Aut $\pi)$ if there exists a $\theta$ isomorphism $\beta: M \rightarrow M^{\prime}$. We say that $M$ and $M^{\prime}$ are (Aut $\pi$ )-isomorphic iff $M, M^{\prime}$ are $\theta$-isomorphic for some $\theta \in$ Aut $\pi$; they are stably isomorphic if $M \oplus(Z \pi)^{i} \cong M^{\prime} \oplus(Z \pi)^{i}$ for some $i \geqslant 0$. Here isomorphism ("œ") means idisomorphism. Let ${ }^{*}{ }_{M}$ denote the class of $\pi$-modules $M^{\prime}$ which are (Aut $\pi$ )- 
isomorphic to $M$. A module $M^{\prime}$ is a noncancellation example for $M$ iff (a) $M$ and $M^{\prime}$ are stably isomorphic and (b) $M, M^{\prime}$ are not (Aut $\pi$ )-isomorphic. Let $\Re \mathcal{C}(M)$ denote the set of (Aut $\pi$ )-isomorphism classes of noncancellation examples of $M$ together with ${ }^{*} M$. Thus $\pi$-modules $M^{1}$ amd $M^{2}$ belong to the same class in $\Re \mathcal{C}(M)$ iff $M$ and $M^{i}$ are stably isomorphic $(i=1,2)$ and $M^{1}$, $M^{2}$ are (Aut $\pi$ )-isomorphic.

It happens in some cases that $\Re \mathscr{C}(M)$ is a group [3]. For example, if $\pi$ is finite and $M$ is $Z \oplus Z \pi$ ( $Z$ has the trivial module structure) then $\vartheta \bigodot(Z \oplus Z \pi)$ is isomorphic to a quotient group of a subgroup of $\operatorname{Pic}(Z \pi)$.

1.4. Corollary. Let $\vec{\chi}: H T(\pi, m) \rightarrow Z$ be given by $\vec{\chi}[X]=(-1)^{m} \chi(X)$, where $\chi(X)$ is the Euler character of the $(\pi, m)$-complex $X$. Under the hypotheses of 1.2 , the set of homotopy types of $(\pi, m)$-complexes $X$ having the same directed Euler character $\vec{\chi}[X]=i$ is given by $\vec{\chi}^{-1}(i)=\Re \mathcal{C}\left(\pi_{m}(X)\right)$.

2. $e^{n}$-groups.

DEFINITION. A group $\pi$ has property $e^{n}(n \geqslant 0)$ iff:

(a) For each free $\pi$-module $F, H^{i}(\pi ; F)=0$ for $i \neq n$.

(b) For each group $G$ having $\pi$ as a normal subgroup, let $F^{\prime}$ be an arbitrary free $G$-module. Then $H^{n}\left(\pi, F^{\prime}\right) \cong Z \otimes_{Z \pi} F^{\prime}$ as a $G / \pi$-module, where $Z$ is the trivial $G$-module.

2.1. Proposition. (i) $\pi$ is a $e^{0}$-group iff $\pi$ is finite.

(ii) Any Poincaré duality group (PD-group) having cohomological dimension $n$ is a $\mathrm{e}^{n}$-group.

Proof. (i) If $\pi$ is an infinite $e^{0}$-group, then by (b), $H^{0}(\pi ; Z \pi) \cong Z$ which is impossible. For $\pi$ finite, it is well known that (a) is true because $Z \pi$ is weakly injective. To see (b), let $\pi \triangleleft G$ and $F^{\prime}$ be a free $G$-module. Then $H^{0}\left(\pi, F^{\prime}\right)$ $\cong\left(F^{\prime}\right)^{\pi} \cong Z \otimes_{Z \pi} F^{\prime}$ as $G / \pi$-modules.

(ii) For $\pi$ a Poincaré duality group of cohomological dimension $n$, (a) follows from Theorem 2.3 of [2] and (b) from Lemma 2.3 of [1].

Now let $e$ be the class of groups $\pi$ which are $e^{n}$ for some $n \geqslant 0$. $e$ is not closed under sums (see [5, p. 138]).

\subsection{Proposition. $e$ is closed under extensions.}

We say that a group $G$ is a poly $(e)$-group if there exists a finite sequence of subgroups

$$
G=G_{0} \triangleleft G_{1} \triangleleft \cdots \triangleleft G_{n-1} \triangleleft G_{n}=1
$$

such that each $G_{i} / G_{i+1}$ is a $\operatorname{group}(i=0, \ldots, n-1)$.

\subsection{Corollary. Any poly (e)-group is a e-group.}

For example, any poly cyclic group is a $e^{n}$-group, where $n$ is the Hirsch number of the group [1]. By 2.1, any poly (PD or finite) group is a -group; similarly for any poly (finitely generated abelian) group.

Proof OF 2.2. Let $1 \rightarrow \pi_{1} \rightarrow G \rightarrow \pi_{2} \rightarrow 1$ be an extension of a $e^{n}$-group $\pi_{1}$ by a $e^{m}$-group $\pi_{2}$. Consider the Lyndon-Hochschild-Serre spectral sequence with abutment $H^{i}(G ; F)$ and $E_{2}$-term $E_{2}^{p, q}=H^{p}\left(\pi_{2}, H^{q}\left(\pi_{1}, F\right)\right)$ where $F$ is a free $G$-module. $F$ is also a free $\pi_{1}$-module, so 


$$
H^{q}\left(\pi_{1}, F\right)= \begin{cases}0 & (i \neq n), \\ Z \otimes_{Z \pi_{1}} F & (i=n) .\end{cases}
$$

But $Z \otimes_{Z \pi_{1}} F$ is a free $\pi_{2}$-module and hence $E_{2}^{p, q}=0$ unless $(p, q)=(m, n)$. Thus $H^{i}(G, F)=0$ unless $i=m+n$ and (a) is satisfied.

To show (b) let $G \triangleleft G^{\prime}$. Then we have the following commutative exact diagram of groups and homomorphismș:

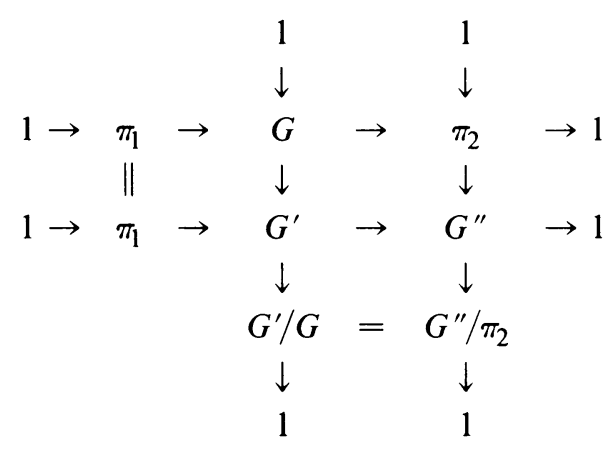

It follows that $\pi_{1} \triangleleft G^{\prime}$ and $\pi_{2} \triangleleft G^{\prime \prime}=G^{\prime} / \pi_{1}$. Let $F^{\prime}$ be any free $G^{\prime}$-module. Then

$$
H^{m+n}\left(G, F^{\prime}\right) \cong H^{n}\left(\pi_{2}, H^{m}\left(\pi_{1}, F^{\prime}\right)\right) \cong H^{n}\left(\pi_{2}, Z \otimes_{Z \pi_{1}} F^{\prime}\right)
$$

by (b) for $\pi_{1}$. But $Z \otimes_{Z \pi_{1}} F^{\prime}$ is a free $\pi_{2}$-module and hence

$$
H^{m+n}\left(G ; F^{\prime}\right) \cong Z \otimes_{Z \pi_{2}}\left(Z \otimes_{Z \pi_{1}} F^{\prime}\right) \cong Z \otimes_{Z G} F^{\prime}
$$

as $G^{\prime \prime} / \pi_{2}=G^{\prime} / G$ modules. The last isomorphism follows because

$$
\begin{aligned}
Z \otimes_{Z \pi_{2}}\left(Z \otimes_{Z \pi_{1}} Z G^{\prime}\right) & \cong Z \otimes_{Z \pi_{2}} Z G^{\prime \prime} \cong Z\left(G^{\prime \prime} / \pi_{2}\right) \cong Z\left(G^{\prime} / G\right) \\
& \cong Z \otimes_{Z G} Z\left(G^{\prime}\right)
\end{aligned}
$$

This completes 2.2 .

Suppose a group $G$ contains a $e^{n}$-group $\pi$ as a normal subgroup. Then 2.2 implies that $H^{i}(G, Z G) \cong H^{i-n}(G / \pi ; Z(G / \pi))$ for all $i \geqslant 0$.

Note added in proof: R. Bieri has shown that poly (finite or duality) groups $G$ also have the property that $H^{i}(G ; \mathrm{ZG})=0$ for all but one value of $i$.

\section{BIBLIOGRAPHY}

1. R. Bieri, Gruppen mit Poincaré-Dualität, Comment. Math. Helv. 47 (1972), 373-396.

2. R. Bieri and B. Eckmann, Groups with homological duality generalizing Poincaré duality, Invent. Math. 20 (1973), 103-124. MR 49 \#5204.

3. M. Dyer, Homotopy classification of $(\pi, m)$-complexes. I, II, III (to appear J. Pure and Applied Algebra).

4. - Projective $k$-invariants (to appear).

5. K. Gruenberg, Cohomological topics in group theory, Lecture Notes in Math., vol. 143, Springer-Verlag, New York, 1970. MR 43 \#4923.

6. F. E. A. Johnson and C. T. C. Wall, On groups satisfying Poincaré duality, Ann. of Math. (2) 96 (1972), 592-598. MR 47 \#358.

7. S. Mac Lane and J. H. C. Whithead, On the 3-type of a complex, Proc. Nat. Acad. Sci. U.S.A. 36 (1950), 41-48. MR 11, 450. 